\title{
UAS Mapping as an alternative for land surveying techniques?
}

Lomme Devriendt, Johan Bonne

Orbit GeoSpatial Technologies

Photogrammetry Department

Scherpeputstraat 14

9160 Lokeren

Belgium

lomme.devriendt@orbitgis.com, johan.bonne@orbitgis.com

Key Words: UAS Mapping, Stereo Photogrammetry, low-altitude high-precision mapping

Can a UAS mapping technique compete with standard surveying techniques? Since the boom in different RPAS (remotely piloted air system), UAV (unmanned aerial vehicle), or UAS (unmanned aerial system), this is one of the crucial questions when it comes to UAS mappings. Not the looks and feels are important but the reliability, ease-to-use, and accuracy that you get with a system based on hardware and corresponding software. This was also one of the issues that the Dutch Land Registry asked a few months ago aimed at achieving an effective and usable system for updating property boundaries in new-build districts. Orbit GT gave them a readymade answer: a definitive outcome based on years of research and development in UAS mapping technology and software.

Although conventional surveying techniques still remain in place, it is a fact that under certain circumstances it is of far greater interest to focus on UAS measurements. For a number of years now Orbit GeoSpatial Technologies have been conducting highly targeted turnkey projects involving the land surveying application of UASs, concentrating on quality, accuracy and reliability. Using UASs means that places that a surveyor finds hard to reach, construction sites subject to vibrations, vertical surveys for heritage restoration projects, large-volume calculations on infrastructure works, areas where photographic material provides clear added value and areas where there are high buildings or bulky infrastructure, no longer present an insuperable problem. Using modern UAS mapping technology, a resolution of $1 \mathrm{~cm}$ and accuracy of $2 \mathrm{~cm}$ is easy to achieve at a cost that is definitely competitive when compared with traditional methods of collecting data.

Although it seems like any conventional photogrammetry packet combined with any UAS system will end in satisfying results, there is a big difference between nice visualizations and land surveying quality. To tackle this quality gap, Orbit GeoSpatial Technologies has decided some years ago, although every airborne system is still supported in the software, to go more deeply into one specific UAS system which is most suitable for accurate data captation. Integrating this UAS system with specialized pre- and postflight software, that feeds the preprogrammed waypoint flight in a 'photogrammetry' way and adapts the postflight process on required image and flightlog data, results in a never seen high accuracy... ready to use and proved for real land surveying tasks.

0/ The assignment from the Dutch Land Registry: to survey the new-build districs in Nunspeet, the Netherlands 
A test area in a new-build district located the borough of Nunspeet (Netherlands) with visible boundaries (hedges, fences, walls, building, etc.) on the ground was nominated for a challenge. The aim of the test was to develop a method by which the boundaries of the individual plots could be shown on an aerial photograph and then be used officially afterwards via stereo mapping. Before the flight, the Land Registry surveyed a number of plotting and check points. The aerial photos are now being used for additional research or for placing photographic material online, providing owners with a simple, unambiguous indication of their boundaries and demonstrating whether the accuracy of stereo mapping enables the data recorded to be included subsequently as part of official surveys.

In what follows, we run through the process and discuss the basic principles of the Orbit UAS mapping solution: (1) the Orbit preflight software, (2) the MD4-1000 system and (3) the Orbit postflight software. Hereafter (4), we end by some conclusions and extra integrated values of this farreaching UAS mapping solution.

\section{1/ Flight planning and recording with Orbit UMP software}

Using the Orbit UMP (UAV Mapping Planner) software, a flight plan for the test was easily drafted in a user friendly GIS environment using a minimum number of steps. The here-employed settings for this new-build district test included (1) type of UAS (MD4-1000), (2) camera mounting and specifications (Olympus EP3 camera with a focal of 17mm), (3) flight area (1.5ha) and flight direction (along the road for optimal coverage), and (4) the parameters for achieving the best possible stereo coverage at a certain height. The flight plan set out the coordinates (waypoints) for the fully automatic UAS flight complying an accurate stereo recording: flying height above ground level was about $58 \mathrm{~m}$ which results in a ground pixel size of $1.5 \mathrm{~cm}$. The foto-overlap was influenced by the Dutch Land Registry and resulted in a 80\%-80\% overlap for different tests. Number of photos taken was 196 in a flight time of 19.03 minutes covering an area of about 1.5 ha.

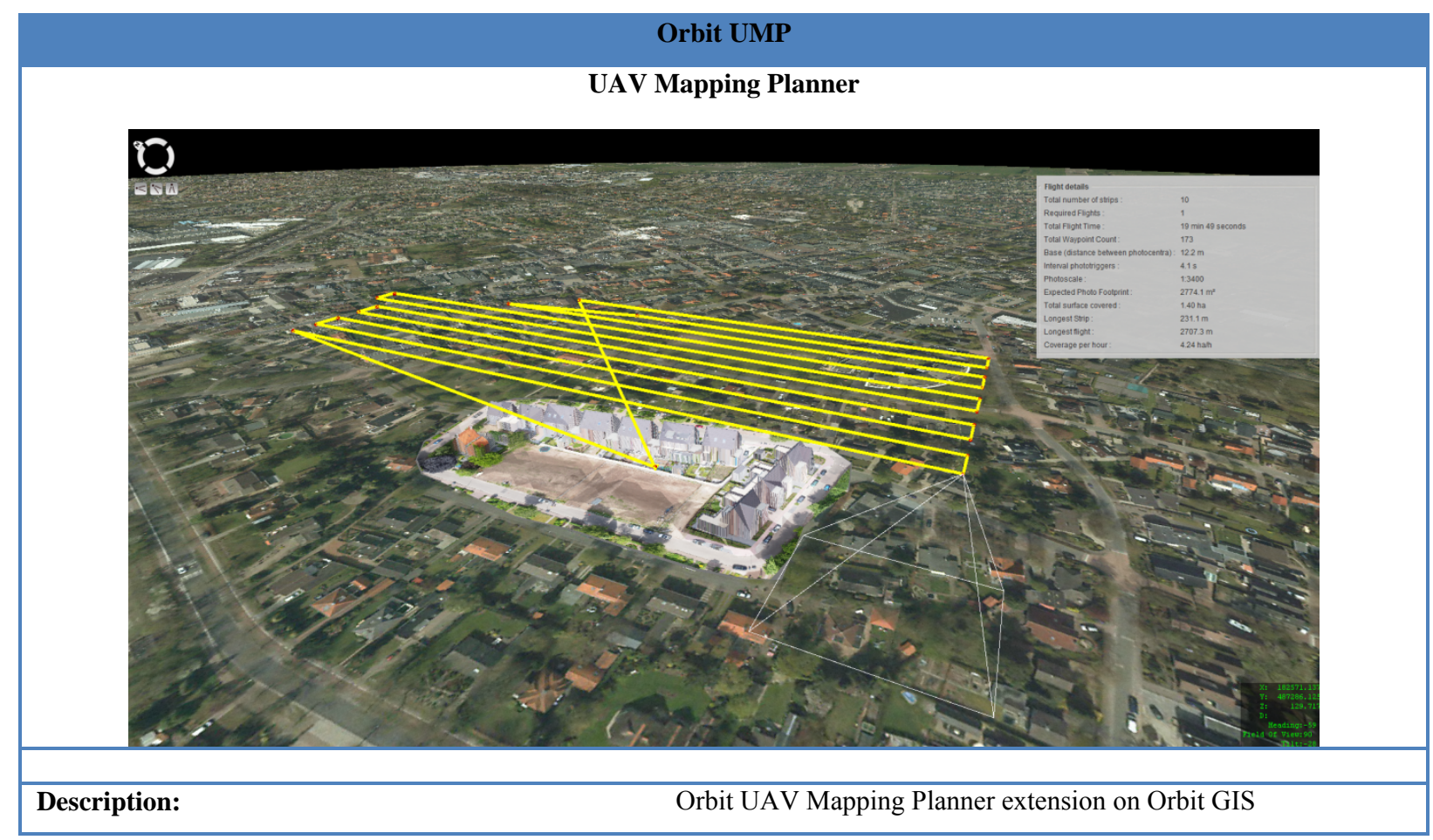


The International Archives of the Photogrammetry, Remote Sensing and Spatial Information Sciences, Volume XL-3/W1, 2014 EuroCOW 2014, the European Calibration and Orientation Workshop, 12-14 February 2014, Castelldefels, Spain

\begin{tabular}{|ll|}
\hline UAS system & Specifications \\
\hline Payload & MD4-200 and MD4-1000 \\
\hline Time to prepare flight plan & $\begin{array}{l}\text { Supports all types of cameras (tested for mapping: Olympus EP3, } \\
\text { Sony Nex-7, Canon 550D-660D, Canon MarkIII) }\end{array}$ \\
\hline Number of steps to taken & $<5$ minutes, GIS integrated \\
\hline & $\begin{array}{l}\text { 1. UAS settings } \\
\text { 2. Payload settings }\end{array}$ \\
& 3. Area to cover \& flight direction \\
& 4. parameters for stereocoverage \\
\hline Flight plan & Split up in different flights (depending on area) \\
& Ready-to-use for UAS import \\
\hline
\end{tabular}

\begin{tabular}{|ll|}
\hline Sample: & MD4-1000 + Canon550D payload \\
\hline Flight height above ground level & $70 \mathrm{~m}$ \\
Ground Pixel Size & $1.2 \mathrm{~cm}$ \\
Overlap & $60 / 30 \%$ \\
Continuous flight & yes \\
\hline Coverage per hour & $28.79 \mathrm{ha} / \mathrm{h}$ \\
photoscale & $1 / 2900$ \\
\hline
\end{tabular}

\section{2/The Microdrone: a UAS suitable for accurate data captation}

With the assistance of the most professional UAS on the market, a usable and accurate solution is provided for surveying and updating map material. Putting the Microdrones MD4-1000 to work for mapping purposes shows some remarkable advantages compared to conventional techniques in aerial photogrammetry. Very important properties of this UAS are the ease to operate, the extreme flight time, the professional reliability, and overall usability. In contrast to other UAS systems the professionalism and long research done on this system results in high reliable operations needed for today's UAS legislations. Furthermore, the 6 year collaboration between Orbit GT and Microdrones made this drone extreme useful for mapping purposes: the MD4-1000 is the only device that truly meets the standard requirements imposed by photogrammetry. Moreover, the choice of four relatively large propellers results not only in a very low noise level (68dBA at a distance of $3 \mathrm{~m}$ ) but even more important in an extreme long flight time as a results of the intelligent power distribution. Since the stability and reliability of the UAS is controlled automatically using a number of sensors and sophisticated firmware, and since a preprogrammed GPS flight plan can be performed fully autonomous as well as aerial imagery taken on optimized and precalculated positions, one operator can easily perform any task with minimum of efforts in almost all weather circumstances.

\section{md4-1000}

\section{Microdrones}




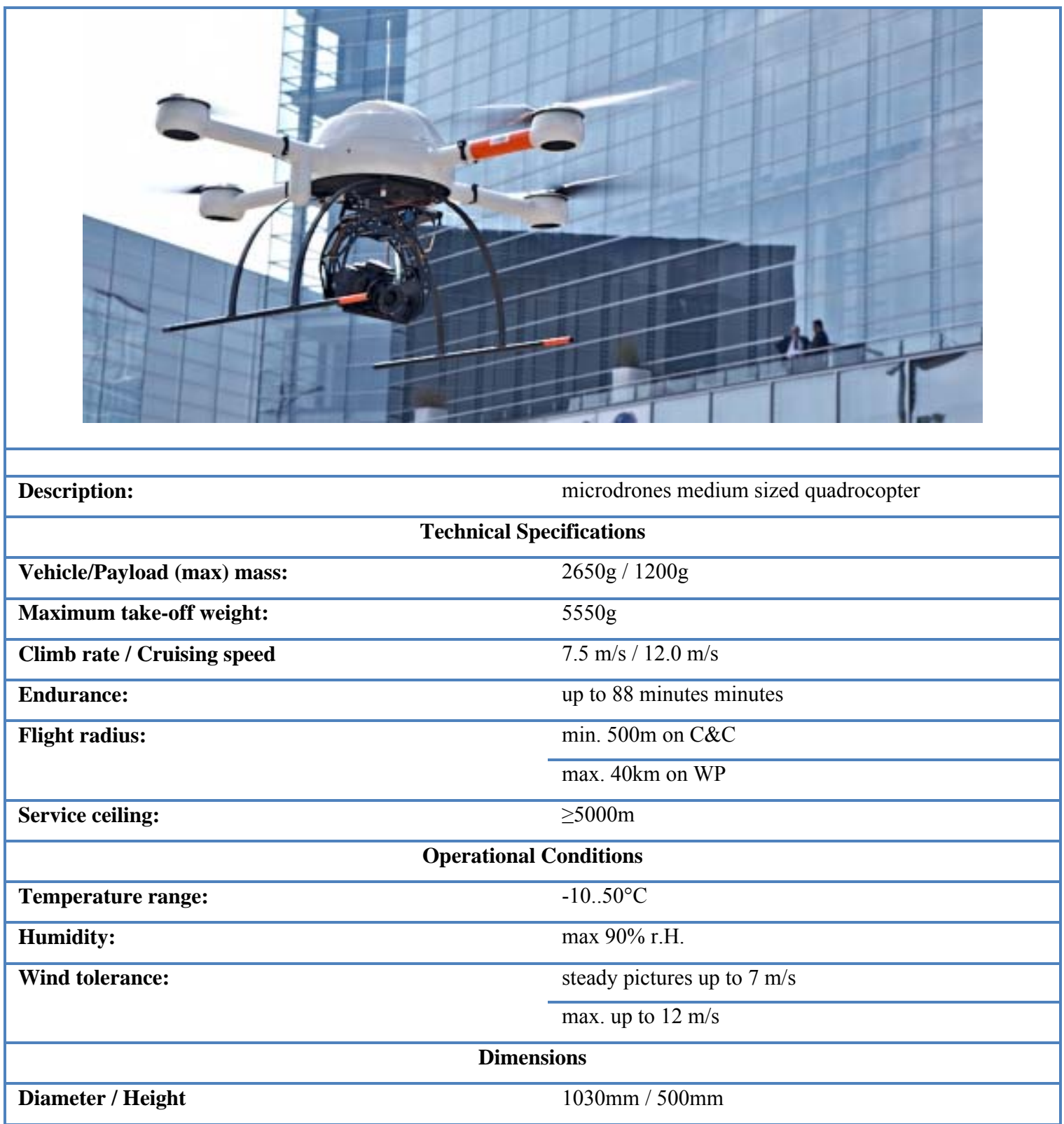

\section{3/Data-processing with Orbit Strabo Software}

After a flight lasting just 19 minutes to survey an area of approximately 1.5 hectares, the drone landed safely again. As it was a project following in the wake of two failed flights using other UASs, this was finally a short and successful mission carried out by Orbit GT. After loading the images and external orientation file from the MD4-1000, the project - based on a fully automatic plotting point and matching procedure - was completed in no time. The results speak for themselves: the error in $\mathrm{x}$ and $\mathrm{y}$ on object points (and ground reference points) is better than expected. The mean squared error for the matched object points are almost identical to the pixel resolution of $1.5 \mathrm{~cm}(\mathrm{z}=2-3 \mathrm{x})$. This is certainly a level of resolution considered to be sufficient for surveying and if required can be refined further by setting a lower flight altitude or by using a different type of camera or lens (e.g. Canon 550D or Sony Nex-7). 
The International Archives of the Photogrammetry, Remote Sensing and Spatial Information Sciences, Volume XL-3/W1, 2014 EuroCOW 2014, the European Calibration and Orientation Workshop, 12-14 February 2014, Castelldefels, Spain

\begin{tabular}{|llll|}
\hline & msqrX & msqrY & msqrZ \\
\hline Mean Squared Error for object coordinates (m): & 0.017 & 0.014 & 0.054 \\
\hline Average MSQE for object coordinates $(\mathbf{m}):$ & 0.014 & 0.013 & 0.048 \\
\hline Standard deviation for object coordinates (m): & 0.021 & 0.013 & 0.056 \\
\hline & & & $m s q r Z$ \\
\hline Mean Squared Error for ground control points coordinates (m): & $m s q r X$ & $m s q r Y$ & $m s$ \\
\hline Average MSQE for ground control points coordinates (m): & 0.007 & 0.015 & 0.036 \\
\hline Standard deviation for ground control points coordinates (m): & -0.002 & -0.001 & -0.001 \\
\hline
\end{tabular}

\section{$\underline{\text { 4/ 3D visualisation and stereo mapping with Orbit Strabo. }}$}

However, it is not just the speed and accuracy of the fully automatic adjustment that springs to mind, but the 3D visualization and totally automatic DTM (Digital Terrain Model) creation make other applications achievable in practice. by adding breaklines via stereo mapping to the DTM, proper 3D visualization and accurate true orthophotos become available, which produce some fine shots.

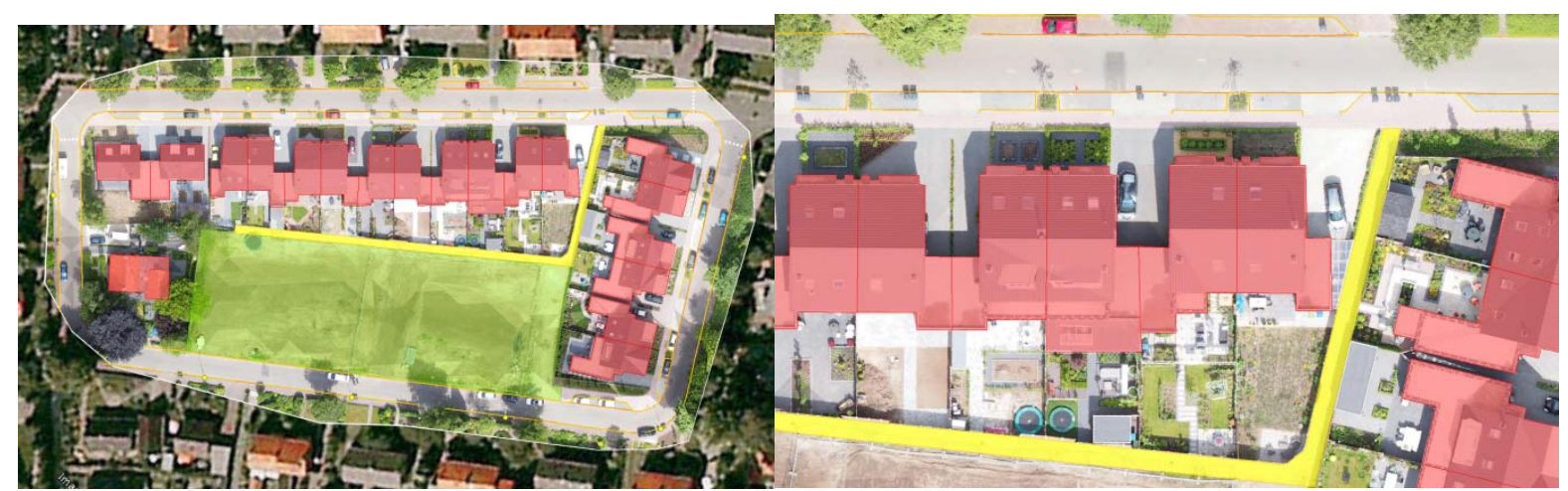

The accuracy of stereo recording - based on a fixed flight plan and made using a professional UAS means that mapping in stereo images becomes possible. As a result and using anaglyphs, stereographics or Orbit's optimized Strabox mode, 3D coordinates can be measured accurately. This enables property boundaries on the ground or any other point, line or surface element to be entered; integrating the Orbit Strabo software into the Orbit GIS package also means adding other attributes into the mix. 


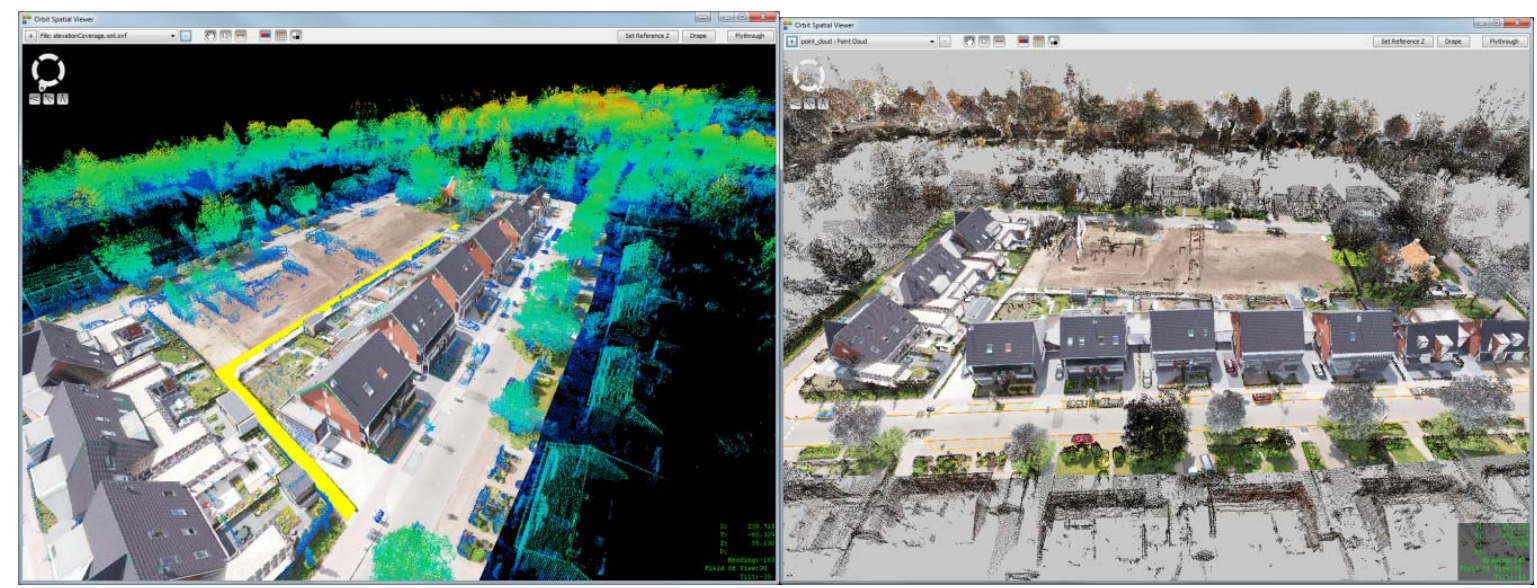

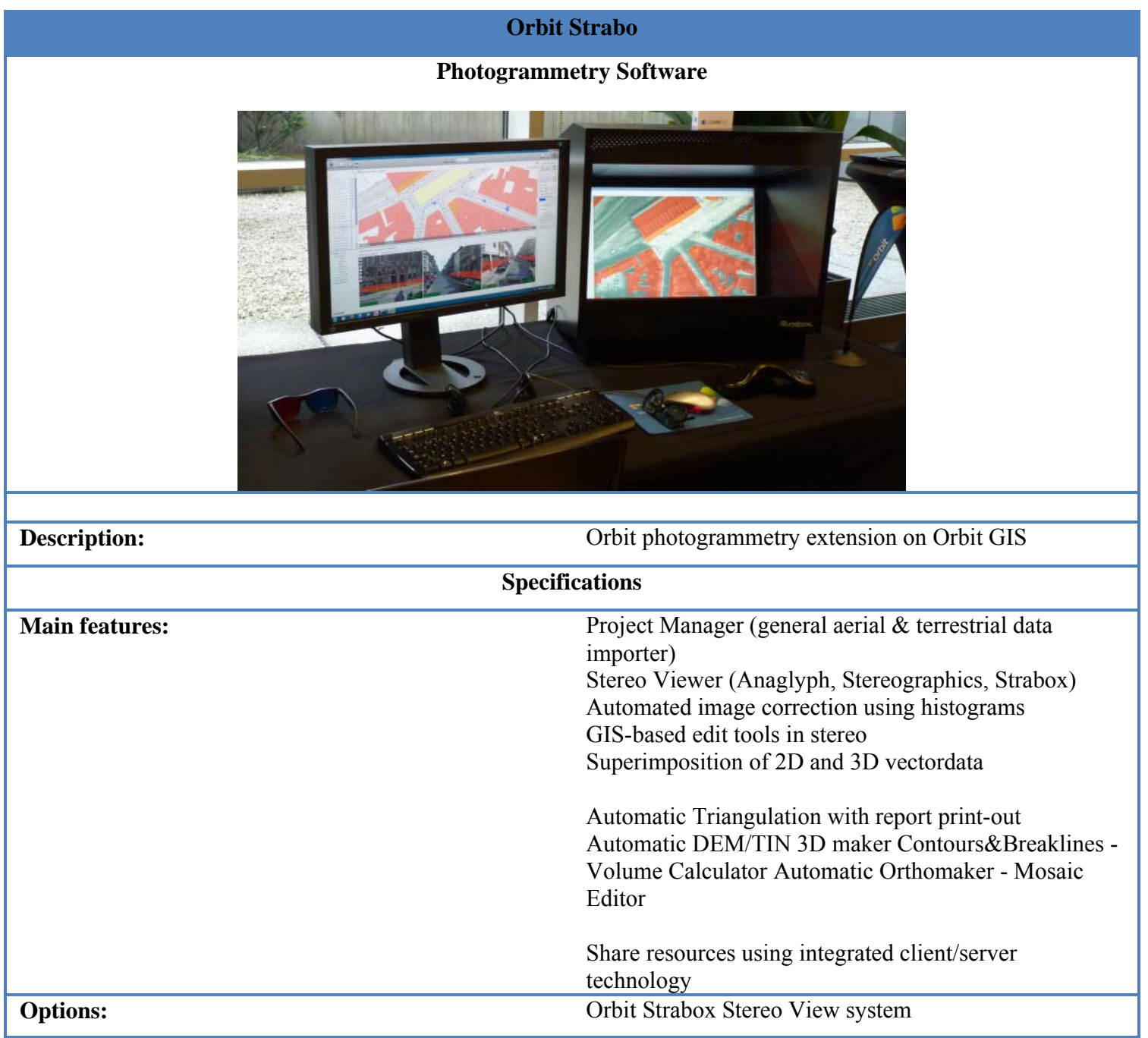

The next step is to use the photographs taken and the height model produced to make a fully automatic, high-resolution 'true' orthophoto. This type of orthophoto is different in that all of the 'lying down' buildings are now standing nice and upright. In addition, the Orbit software pallet also integrates all kind of other geodata such as point cloud data coming from fixed or mobile laserscannings. In the image below we can observe a mobile mapping point cloud from Topcon's IPS2 
integrated with the here-presented UAS imagery and restituted vector data. This integration finally brings together all photogrammetry and laserscan data combining the benefits of both.

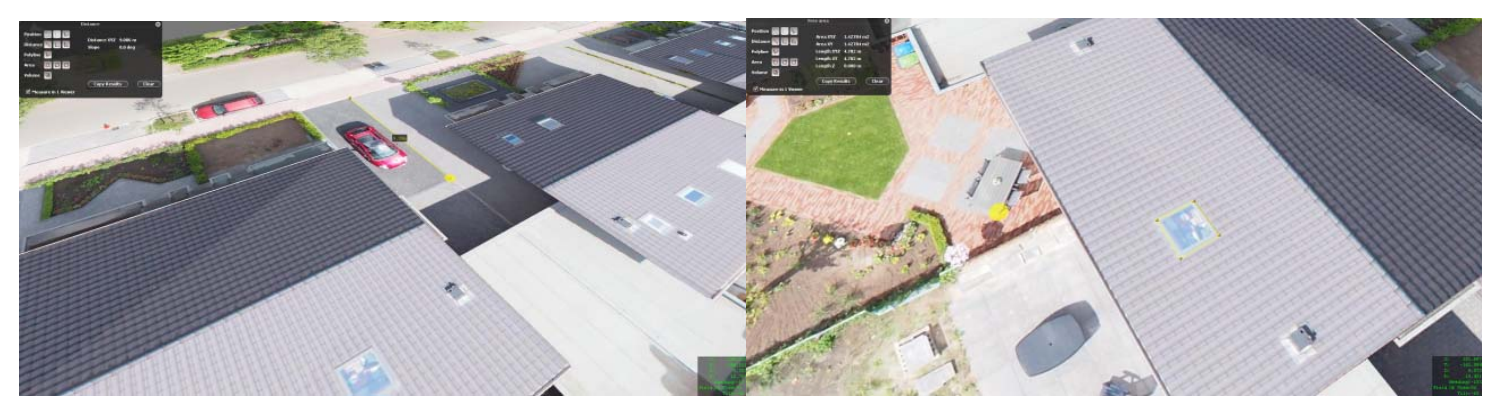

Although the UAS technology presented here is not likely to replace surveyors and their theodolites any time soon, it does demonstrate that the technology can be used increasingly in specific circumstances. The photographic material produced ensures that this technology stands out clearly from traditional surveying. And it does mean that UAS surveying technology - backed by the results from this pilot - can finally be taken seriously. 